\title{
Design and Application of Insulator Detection Robot System for UHVDC Transmission Line
}

\author{
Xueneng Huang ${ }^{1}$, Yuwen Xie ${ }^{2}$, Chongjie Ma ${ }^{1}$, Jinpeng Tan ${ }^{1}$, Dongfeng Qiu ${ }^{1}$, Youneng Wu ${ }^{1}$, \\ Su Fang ${ }^{3}$, Yitao Lu${ }^{1}$, Yongjian Lin ${ }^{1}$, Xianning $\mathrm{Li}^{1}$ \\ ${ }^{1}$ Wuzhou Bureau of China Southern Power Grid UHV Transmission Company, Wuzhou City, China \\ ${ }^{2}$ Beijing Guowang Fuda Science \& Technology Development Co. Ltd, Beijing City, China \\ ${ }^{3}$ Maintenance Test Center of China Southern Power Grid UHV Transmission Company, Guangzhou City, China
}

Email address:

454962380@qq.com (Xueneng Huang)

\section{To cite this article:}

Xueneng Huang, Yuwen Xie, Chongjie Ma, Jinpeng Tan, Dongfeng Qiu, Youneng Wu, Su Fang, Yitao Lu, Yongjian Lin, Xianning Li. Design and Application of Insulator Detection Robot System for UHVDC Transmission Line. International Journal of Mechanical Engineering and Applications. Vol. 7, No. 4, 2019, pp. 106-110. doi: 10.11648/j.ijmea.20190704.13

Received: June 15, 2019; Accepted: August 16, 2019; Published: September 4, 2019

\begin{abstract}
In recent years, with the vigorous construction of the ultra-high voltage grid, the line operating voltage level has become higher and higher. In order to ensure the safe operation of the transmission line, the line insulator string has a length of several tens of meters, and traditional manual testing has been unable to meet the requirements. With the continuous maturity of wireless communication technology, electromagnetic compatibility technology, sensor technology and other comprehensive technologies, it is necessary to develop an insulator string detection robot to replace artificial insulator detection work on UHV transmission lines. By means of wireless sensor communication technology, electromagnetic compatibility technology, video image technology and many other technologies, the insulator detection robot designed in this paper has realized automatic detection of zero resistance value of insulator of UHV transmission line. In addition, the real-time monitoring of insulator resistance value can be carried out through wireless communication to achieve the purpose of timely alarm. In addition, the robot's motion status and the insulator's field status can be observed in real time through video image acquisition. The insulator detection robot designed in this paper realizes the automatic detection of the zero value of insulator of the UHV DC transmission line. It can adapt to the UHV AC DC transmission line with different voltage levels by changing the length of the climbing arm, and at the same time adapt to the climbing detection of the string insulator of tension tower and the vertical insulator of the straight tower. The problem that the insulator string of the UHVDC transmission line can not be automatically detected by electric charge has been solved. It greatly improves the efficiency of insulator detection and the automation level of power transportation maintenance, reduces the cost of human transportation, and creates higher economic and social benefits.
\end{abstract}

Keywords: Insulator Detection, Robot, Ultra High Voltage DC, STM32

\section{Introduction}

As an important part of overhead transmission lines, insulators' insulation performance is directly related to the safe operation of transmission lines. Due to the influence of external environment such as pollution, the performance of insulators will decline, In recent years, with the vigorous construction of UHV power grid, the operating voltage level of transmission lines is getting higher and higher, in order to ensure the safe operation of transmission lines, line insulator strings are tens of meters long, and the traditional manual detection has been unable to meet the requirements.

With the development of wireless communication technology, electromagnetic compatibility technology, sensor technology and other integrated technologies, it is necessary to develop an insulator string detection robot to replace manual inspection of insulators on UHV transmission lines. Automatic inspection by robots can not only solve the problem of live detection of insulator strings in UHV transmission lines, improve the efficiency and accuracy of inspection, but also reduce the cost of personnel, eliminating hidden dangers such as falling at high altitude and electric shock of personnel. [1, 2] 


\section{Method}

\subsection{System Design}

The insulator detection robot designed in this paper realizes the zero value automatic detection of insulator resistance of UHV transmission line by using wireless sensor communication technology, electromagnetic compatibility technology, video image technology and other technologies. The real-time monitoring of insulator resistance can be achieved through wireless communication to achieve the purpose of timely alarm. In addition, real-time observation can be achieved through video image acquisition. The motion state of the robot and the field condition of the insulator and the product diagram is shown in Figure 1.

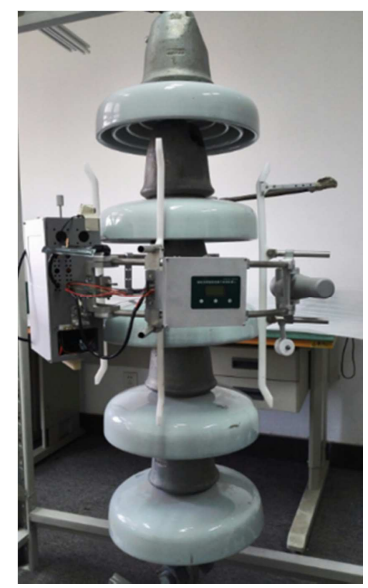

Figure 1. Insulator Inspection Robot Product Diagram.

\subsection{Mechanical Mechanism Design}

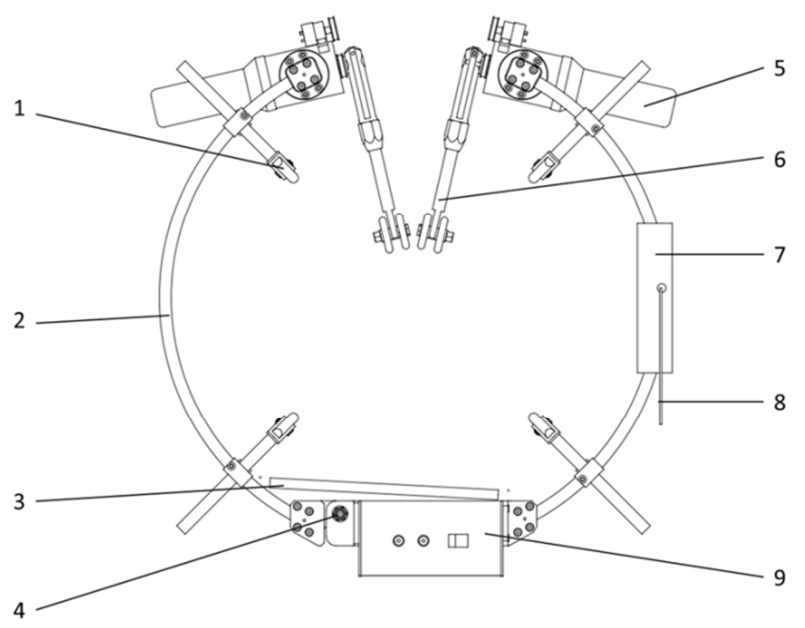

Figure 2. Mechanical Structure Design of Insulator Inspection Robot.

1. Main guide rod. 2. Walking arm connecting bracket. 3. Elastic protection mechanism. 4. Elastic Rotating Axis. 5. Executive motor. 6. Walking arm. 7. Detection module. 8. Test pen. 9. Control cabinet.

The robot is designed with a climbing intelligent insulator detection robot with one side and two rotating arms. Insulator string intelligent detection robot can walk on the insulator string by itself, and inspect the electrical performance of the insulator piece by piece according to the requirements, thus replacing part of manual operation, improving safety and work efficiency. The external dimension of the scheme also has the characteristics of compact structure, easy to carry and easy to use. The mechanical structure design is shown in Figure 2.

The structure of single-side double rotating arms can save a lot of space for mechanical movement. Using double-layer circular walking arm to connect the bracket, on the one hand, it can make the walking arm, guide rod, control cabinet, insulation detection module connected to the bracket more stable, without deflection. On the other hand, the circular support is more conformable to the shape of insulator strings in structure and saves the space for motion. Especially in the detection of double insulator strings, it avoids the motion interference of the robot to the adjacent insulator strings in the process of motion.

\subsection{Design of Electrical System}

\subsubsection{Control System Design}

Because the power of the system is supplied by $24 \mathrm{~V}$ batteries, the power consumption of the system is directly related to the working time of the robot. For this reason, the controller of the robot adopts STM32L series products. Based on the ultra-low power core of ARM Cortex-M4 processor, two energy-saving technologies unique to Italian Semiconductor are adopted: $130 \mathrm{~nm}$ special low leakage current manufacturing process and optimized energy-saving architecture to provide industry-leading energy-saving performance.

Robot performance indicators are shown in Table 1.

Table 1. Insulator Inspection Robot Technical Indicators.

\begin{tabular}{ll}
\hline Working environment & $\begin{array}{l}\text { High Voltage Porcelain Insulator Strings } \\
(+800 \mathrm{KV}) \text { and Above }\end{array}$ \\
& Insulator esistance Value Detection \\
Product function & $\leq 10 \%$ \\
measurement accuracy & $\geq 5 \mathrm{~h}$ \\
Homework hours & $\geq 0.03 \mathrm{~m} / \mathrm{s}$ \\
Moving speed &
\end{tabular}

Motor and motor driver are the key components of the robot, which directly affect the motion performance and operation safety of the robot. Considering the influence of the overall structure size and driving load, the brushless motor is adopted in the design. The rated power is $60 \mathrm{~W}$, the maximum continuous output current is $4 \mathrm{~A}$, the peak current is $18 \mathrm{~A}$, the maximum continuous output load is $60 \mathrm{mNm}$ and the maximum speed is $15000 \mathrm{rpm}$.

The angle feedback system is designed by absolute single-loop encoder with a precision of 4096 bits. In order to improve the reliability of the acquisition, low-pass filtering technology is added to the hardware and average median method is used in the software. The electrical system design is shown in Figure 3. The MCU obtains the precise position of the manipulator through real-time angle detection by the encoder, and then drives the motor to climb accordingly. The ground control terminal of the wireless communication system interacts with the robot to obtain the resistance value of 
insulator string. In addition, the three-dimensional motion position of the robot and the surface condition of insulator can be acquired in real time by the video acquisition system.

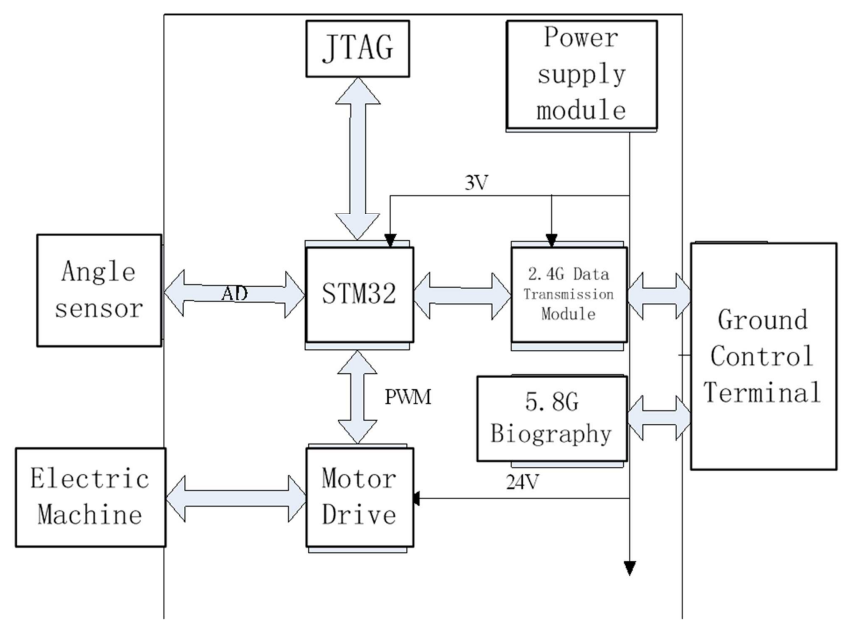

Figure 3. Electrical System Framework.

\subsubsection{Electrical System Design}

\section{Design of Wireless Communication System}

The wireless communication system of the robot adopts 2.4GW WiFi module. The selected integrated WiFi module supports $802.11 \mathrm{~b} / \mathrm{g} / \mathrm{n}$ wireless standard, TCP/IP/UDP network protocol stack and transmission band is $2.412 \mathrm{GHz}-2.484$ $\mathrm{GHHz}$. Because of the serious electromagnetic interference of UHVDC transmission line, in order to avoid the influence of UHVDC electric field environment, the $2.4 \mathrm{GW}$ modules with transmission power of $1 \mathrm{~W}$ is selected in the experiment, which is barrier-free. The communication distance can reach $250 \mathrm{~m}$, which can effectively reduce the impact and ensure the reliability of data transmission. The real-time data collected by the robot and the control instructions of the ground control terminal can interact through the WIFI module.

Design of Video Transmission Module

This video module chooses a camera with low power consumption, small size, light weight, easy installation, high-definition CCD and 360-degree adjustable angle. The camera module is installed on the left and right sides of the robot body and opposite to the control cabinet. The three cameras installed can observe the movement of the insulator and the robot from any angle to judge whether the detected insulator is damaged, cracked and the steel cap pulled out.

The video captured by the camera is sent to the handheld operator (tablet or notebook computer) through the wireless video transmission module. The $5.8 \mathrm{G}$ wireless video transmission module is chosen in this paper. The transmitter is TS832, and the receiver is RC832. It has the characteristics of light weight, low power consumption, convenient installation, safe, stable and reliable data transmission.

\subsubsection{Design of Insulator Detection Module}

Insulator detection module is designed as follows: DC/DC boost circuit module is used to boost $4 \mathrm{~V}$ DC voltage to $2500 \mathrm{~V}$, and metal high voltage detection probe is directly applied to the upper and lower metal fittings of the insulator to be tested.
[3]; The detection system collects the current added to the insulator through the current sensor. The CPU can analyze and calculate the resistance value of the insulator, and transmit the final detection result to the robot processing core STM32 through RS232. In this way, the resistance value of insulator string can be transmitted to the ground control terminal in real time. If the low value insulator is detected, zero value alarm can also be carried out, which is convenient for operation and maintenance personnel to handle. Due to the complex electromagnetic environment of UHVDC transmission lines and various leakage currents on insulators, it is necessary to filter clutter.

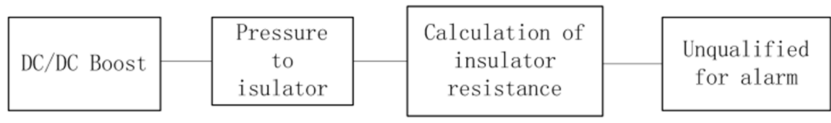

Figure 4. Insulator Detection Logic Architecture.

\subsubsection{Design of Ground Control Terminal}

i. System design

Ground control terminal is designed by $\mathrm{C}++$ programming language under WINDOWS environment. It mainly realizes the functions of robot control command issuance, robot status, insulator resistance, environmental data display, image and video display, etc. Handle operation is specially configured for field us. The architecture is shown in Figure 5.

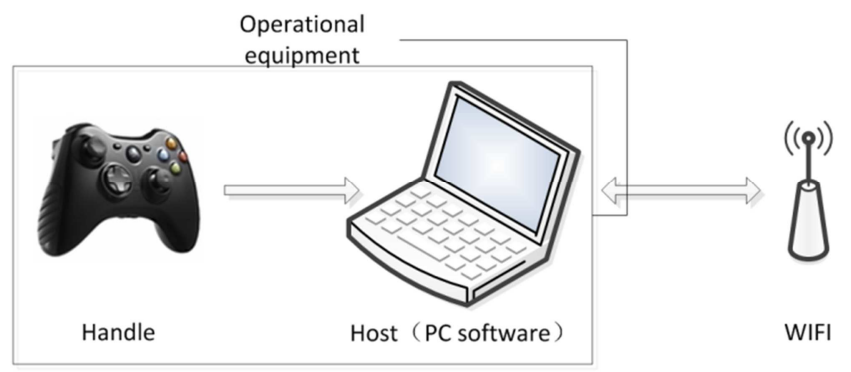

Figure 5. Ground Control Terminal Architecture.

\section{ii. Software Flow Design}

Firstly, when the program starts, the host Registry will be read automatically, the handlebar device and SOCKET communication connection robot will be started, and the device will be initialized. When the staff member chooses the device to use, turn on WiFi and set the timer. In each time cycle, the upper computer software needs to work in two parts at the same time, one is data transmission, the main function is to send action commands to the robot; the second part is data reception, the function is to read the current state parameters of the robot, and real-time display on the software interface. After the work is completed, the interrupt processing flow is opened, the devices are closed, the default values of parameters are restored, and the status is displayed and reset. 


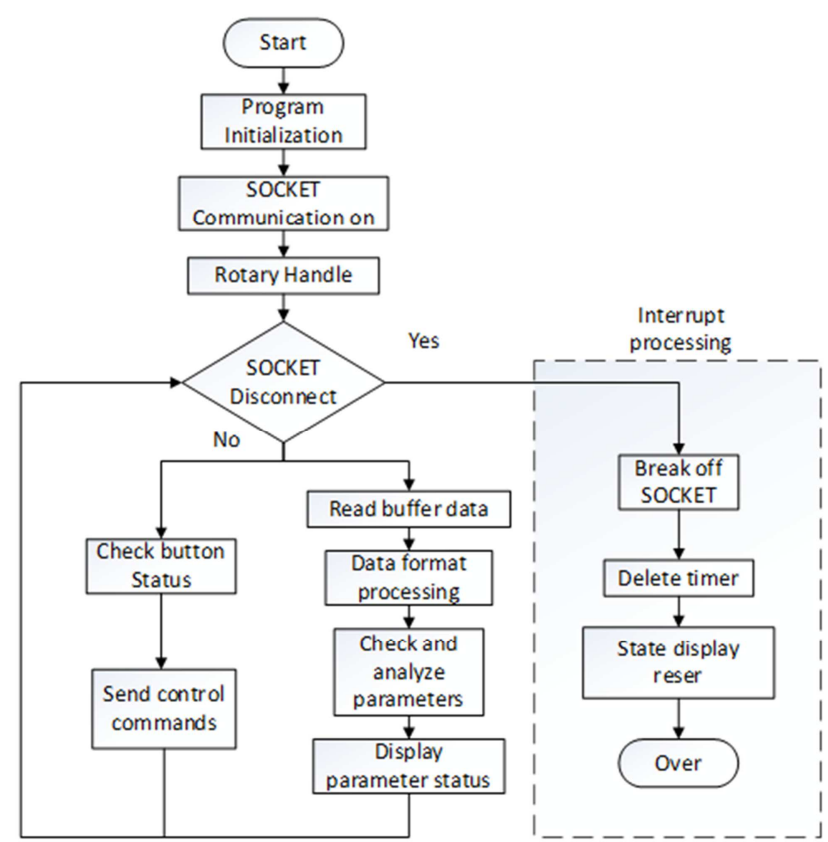

Figure 6. Flow chart of ground control terminal.

\section{Result}

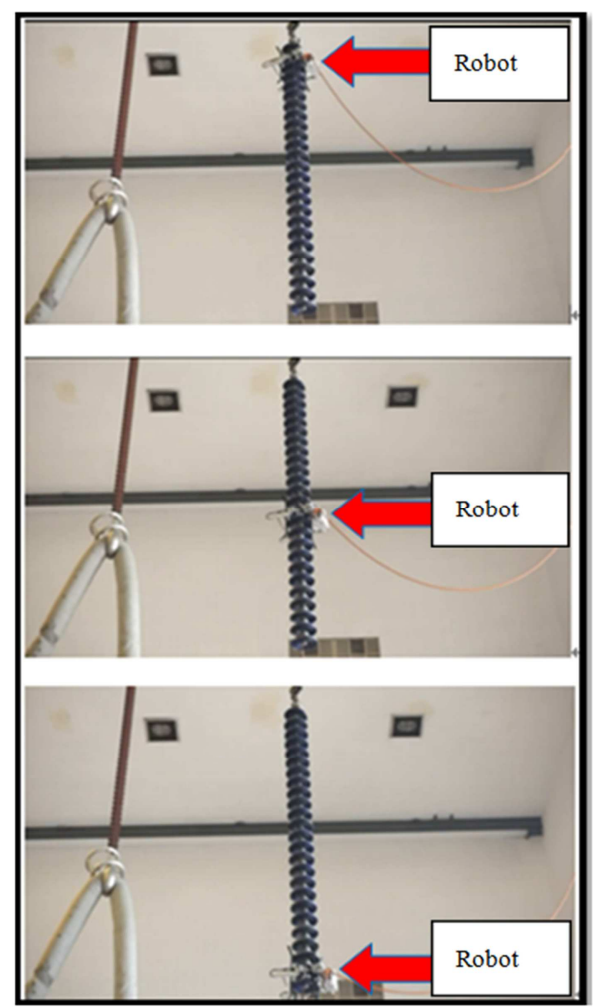

Figure 7. Insulator Inspection Robot $800 \mathrm{kV}$ live Test.

In order to ensure the practicability and reliability of the product, the insulator detection robot has carried out many live tests in the laboratory and on the $(+800 \mathrm{kV})$ Puqiao UHVDC transmission line. The field diagrams of the tests are shown in figures 7 and 8 , respectively. In the laboratory, $+800 \mathrm{kV}$ live test was carried out. The type of insulator is NGK 550KN M-E.
In the laboratory environment, 50 insulators are connected into vertical strings perpendicular to the ground to simulate the insulator strings on the actual lines. The insulators are fixed with metal fittings at both ends to add voltage to $<800 \mathrm{kV}$. Insulator detection robot first puts the first insulator on the top, then automatically detects the resistance of insulator from top to bottom, and sends the resistance signal of insulator detection to the ground control terminal in real time. During the whole experiment, the communication of the robot is normal and the data transmission is accurate.

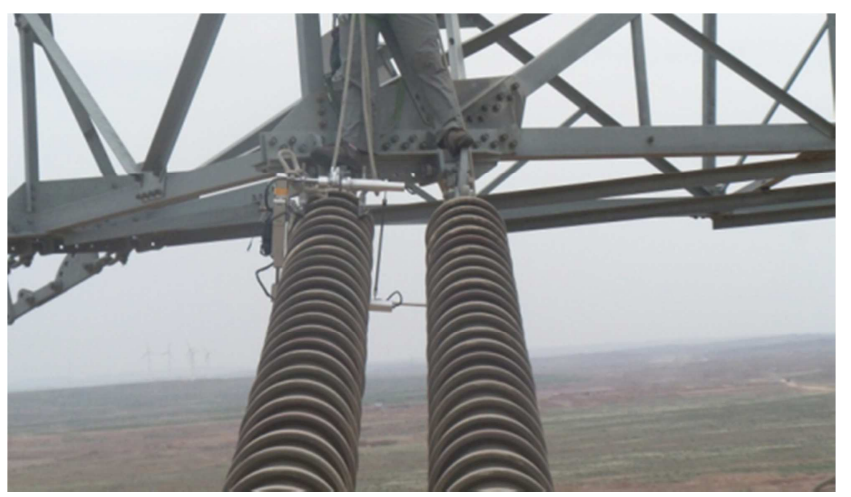

Figure 8. Insulator Inspection Robot Field Test (+800kV).

Insulator detection robot insulation resistance accuracy measurement is calibrated by entrusting Hubei Institute of Measurement Technology. The method of comparing with standard resistance is used to verify the accuracy. As shown in Figure 9, the detection error is less than $1.2 \%$. The design meets the expected requirements and field application.

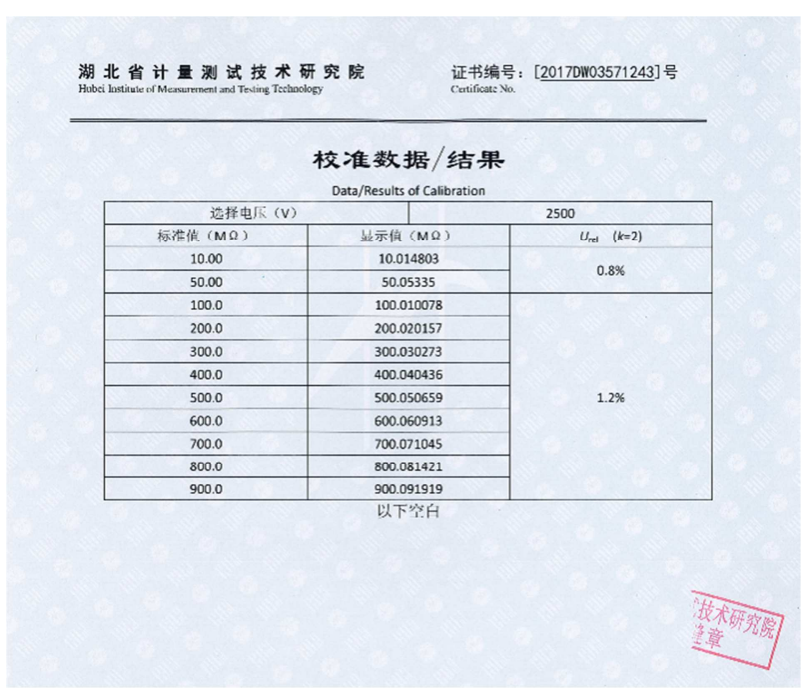

Figure 9. Insulator Inspection Robot Accuracy Test.

\section{Discussion}

The successful design and application of the Insulator Inspection Robot System for UHVDC transmission line fully reflects the superiority of the machine in replacing the personnel. It is expected that in the near future, after batch production, it will serve the vast number of employees in the 
power system and greatly improve the safety and efficiency of power safety production.

\section{Conclusion}

The Insulator Detection Robot designed in this paper realizes the automatic detection of zero value of insulators in UHVDC transmission lines. By changing the length of climbing arm, it can adapt to UHVDC transmission lines of different voltage levels, at the same time, it can adapt to the climbing detection of cross-string insulators of tension tower and vertical-string insulators of straight tower, so as to solve the problem of extra-high insulators. Insulator strings of HVDC transmission lines have always been difficult problems in live automatic detection. It greatly improves the efficiency of insulator detection and the automation level of power operation and maintenance, reduces the cost of manpower operation and maintenance, and creates higher economic and social benefits.

\section{References}

[1] Zhongsheng, Li Taocao, Jun Wang, Daqing Sun, Zhigang Zhao. Design and Research of Intelligent Detection Robot System for Disc-type Porcelain Insulator [J]. Science and Technology and Engineering. 2013 (15).

[2] Dongsong Luo, Jingmei Huang. Insulator Salt Density Online Monitoring System and Its Application [J]. China Electric Power. 2013 (08).

[3] $\mathrm{Hu}$ Zhu, Weiguo Li, Zhi Lin. Current Situation and Development of Insulator Detection Method [J]. Insulators and Surge Arresters. 2006 (06).

[4] Haipeng Shi, Weiguo Li, Zhanzhan Qu. Research on Handheld Insulator On-line Detection Device [J]. Insulators and Surge Arresters. 2012 (01).

[5] Wang Hao. Overview of the development and application of industrial robotics $[\mathrm{J}]$. New technologies and products in China, 2018 (03): 109-110.

[6] Cai Jiyun. Application of Industrial Robot in Automation Control [J]. Science and Technology and Innovation, 2018 (01): 144-145.
[7] Zhao Shaochong. Design and verification of pipelined intelligent robots based on artificial intelligence [J]. China's strategic emerging industries, 2016 (28): 71-75.

[8] Wu Weiguo. Research progress of humanoid robots for operation and artificial intelligence [J]. Journal of Harbin University of Technology, 2015 (7): 1-19.

[9] Cao Zhao. Research and Application of Key Technologies for Compatible Control System of Zhiyun Robot [J]. Innovation and Application of Science and Technology, 2018, (14): 19-21.

[10] Hewett T T, Baccker R, Card S, et al. ACM SIGCHI Curricula for Human-Computer Interaction. New York: ACM, 1992.

[11] Tan Jingyan. Research on the influence of artificial intelligence on interactive design [J]. Packaging Engineering, 2017, 38 (20): 27-31.

[12] Hao Ninghui, Lu Xiaobo. Methodological speculation on the design of entity interaction interface [J]. Decoration, 2014, (2): 34-37.

[13] Shao Changmian. Location and Mapping of Mobile Robots in Dynamic Environment [D] Nanjing University of Posts and Telecommunications, 2014.

[14] Su Qing. Research on path planning and collision avoidance of multi-robots [D] Nanjing University of Posts and Telecommunications, 2014.

[15] Fan Chao; Mechanism Design and Motion Control Simulation of Large Industrial Robot Manipulator [D]; Harbin University of Technology; 2010.

\section{Biography}

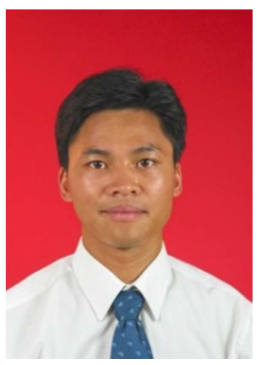

Xueneng Huang (1980-), Senior Engineer, Mainly engaged in UHV and UHV transmission line operation and maintenance, maintenance and safety management. 